\title{
Blonds (Berbères)
}

\author{
G. Boetsch et J.-N. Ferrié
}

\section{OpenEdition}

Journals

Édition électronique

URL : http://journals.openedition.org/encyclopedieberbere/1768

DOI : 10.4000/encyclopedieberbere.1768

ISSN : 2262-7197

\section{Éditeur}

Peeters Publishers

\section{Édition imprimée}

Date de publication : 1 décembre 1991

Pagination : 1539-1544

ISBN : 2-85744-549-0

ISSN : 1015-7344

\section{Référence électronique}

G. Boetsch et J.-N. Ferrié, «Blonds (Berbères) », Encyclopédie berbère [En ligne], 10 | 1991, document B83, mis en ligne le 01 mai 2013, consulté le 24 septembre 2020. URL : http://

journals.openedition.org/encyclopedieberbere/1768; DOI : https://doi.org/10.4000/ encyclopedieberbere.1768

Ce document a été généré automatiquement le 24 septembre 2020.

(C) Tous droits réservés 


\title{
Blonds (Berbères)
}

\author{
G. Boetsch et J.-N. Ferrié
}

1 Contrairement à ce que suggère Pellat dans l'Encyclopédie de l'Islam (1960, p. 1208), l'existence de «berbères blonds" ne saurait être assimilée à un problème supplémentaire, rendant encore plus mystérieuse l'origine des Berbères, dont Gibbon disait déjà qu'elle était «enveloppée dans les ténèbres » (1985, t. II, p. 98). Ainsi que l'indique assez clairement Sergi (1901, p. 59), le problème ethnogénique posé par les «Blonds » est lié à celui de l'origine européenne des Lybiens, c'est-à-dire des Berbères. En fait, la question a été résolue par Kidder, Coon et Briggs (1955, pp. 65-66) qui montrèrent qu'elle n'était pas pertinente, dans la mesure où la tendance au blondisme des populations berbères s'avère équivalente à celle de la plupart des populations méditerranéennes.

2 Néanmoins, plusieurs hypothèses ont été avancées sur l'origine des «Berbères blonds "; ces hypothèses, erronées ou fantaisistes, constituent des représentations actives de l'identité berbère, et interfèrent sensiblement dès qu'il s'agit d'en débattre. $\mathrm{Au}$ demeurant, la plupart d'entre elles, en dépit de ces erreurs, s'inscrivent dans un réel processus d'identification, même si elles demeurent tributaires des aléas scientifiques d'une époque. Aussi importe-t-il de les évoquer avant de s'intéresser aux hypothèses inverses, qui devaient provoquer, sinon leur abandon explicite (ce cas de figure est généralement absent de la vie scientifique), du moins leur oubli partiel.

3 Certes, Vallois affirmera, à son tour, l'existence de blonds nordiques (1944, p. 38), ainsi que Ferembach, qui suggère, en outre, que les Carthaginois, les Romains et les Vandales seraient venus renforcer le contingent des individus à peau claire (1975, p. 116); mais, si l'on cite, aujourd'hui encore, ces origines nordiques, cette thèse ne prête plus à aucun débat scientifique sérieux.

\section{Le paradigme des blonds étrangers}

4 Shaw demeure la référence obligée dès que l'on évoque les «Berbères blonds ». Il les a décrit dans Travels or observations relating to several parts of Barbary and the Levant (1738), faisant des blonds, les descendants des Vandales de Genséric, après qu'ils eussent été 
défaits par Bélissaire : « ces dissemblances nous portent à croire [...] qu'ils sont, sinon la tribu dont parle Procope, du moins les descendants des Vandales » (Shaw, 1830, t. II, pp. 169-170). Buffon, dans son Histoire naturelle de l'homme (1749), cite cette thèse sans donner l'impression d'y adhérer : «ce qui peut faire croire que ces hommes blonds descendent des Vandales" (Buffon, 1792, t. II, p. 84). Gibbon critique directement le point de vue de Shaw, en remarquant que Procope répétant ce que disait le chef maure Orthaïas, affirmait qu'au delà du territoire de celui-ci (situé à l'ouest de l'Aurès) était un vaste désert puis des hommes au corps blanc et à la chevelure blonde. La présence de blonds était ainsi antérieurement attestée à la venue des Vandales. Desmoulin (1826, p. 172) réfutera aussi Shaw, en indiquant qu'il aurait mal interprété Procope. A partir de cette époque, la plupart des auteurs évoquant les blonds, à l'exception notable de Broca (1876, p. 400), se croiront obligés de réfuter la thèse de Shaw comme si personne ne l'avait fait avant eux, tels Vivien de Saint-Martin (1863, pp. 56-57), ou même Gsell (1913, p. 292, note 5), et, plus récemment encore, Leroi-Gourhan et Poirier (1953, p. 115).

5 En fait l'hypothèse vandale s'alimentait à l'idée de peuplements militaires remontant à l'époque romaine (Omalhius d'Alloy, 1859, p. 59). Elle n'est pas loin de l'hypothèse Gauloise évoquée par Féraud (1863, pp. 231-232) à propos des monuments «dits » celtiques de la province de Constantine, où il s'agirait de Gaulois mercenaires ayant introduit leur culte en Afrique, hypothèse reprise une quarantaine d'années plus tard par Saint-Rémy (1904). Une autre hypothèse due à un commentaire de Topinard sur Collignon (Topinard, 1883, p. 12), suggérerait même que les blonds d'Afrique du Nord fussent originaires des Gaulois, mercenaires de Carthage.

6 Il importe de noter que la théorie des blonds, Vandales ou Gaulois, a toujours été utilisée pour expliquer le blondisme d'une population limitée, et n'a jamais été utilisée afin d'expliquer l'origine de la totalité des berbères. Même limité aux kabyles, le blondisme pur est présenté comme la caractéristique d'une tribu; pour le reste, les auteurs favorables à la théorie Vandales font plutôt état d'un métissage (Shaler, 1830 ; Périer, 1873 ; Lenz, 1886 ; Quedenfeld, 1888).

7 L'ensemble des hypothèses celto-nordiques devaient beaucoup aux mégalithes, comme l'indique la position de Féraud. D'une façon générale, l'aspect «celtique » des monuments funéraires a toujours été utilisé comme corroborant la théorie nordique, en tant que traces culturelles d'une migration, qui aurait suivi l'axe nord-est/sud-ouest (Bertrand, 1863). Broca affirmait, par exemple, que «l'origine européenne des blonds d'Afrique septentrionale étant tout aussi certaine que celle des dolmens de la même région, il est tout naturel de penser que ce double fait a été la conséquence d'une seule et même invasion » $(1876$, p. 393).

8 La théorie des blonds du Nord donna également lieu à une hypothèse aryenne, fort controversée au demeurant. Kobelt (1885) pensait ainsi que les aryens blonds avaient précédé les celtes bruns en Afrique du Nord, assertion reprise comme une évidence par Urvoy de Closmadeuc (1898) ou encore Maitrot (1909), qui pensait que les menhirs étaient une manifestation de la race aryenne, et non plus gauloise. On la retrouve affirmée avec force par Lissauer : "Nun wissen wir, dass es nur in Nordeuropa eine Zone gibt, in der eine grosse zusammenhängende blonde Bevölkerung autochton ist, nirgends anders auf der Erde, und sind daher gezwungen anzunehmen, dass auch die sonst auf der Erde inselförmig auftauchenden Blonden von der nordeuropäischen blonden Zone herstammen » " Nous savons maintenant, qu'il n'existe que dans l'Europe 
$\mathrm{du}$ Nord une zone - et nulle part ailleurs - dans laquelle la population blonde est concentrée, et c'est pourquoi nous sommes contraints de penser que les blonds, qui apparaissent isolément sur terre, ont leur origine dans la zone des blonds du Nord " (1908, p. 526). Lissauer pensait, bien sûr, que ces blonds nordiques étaient des Germains, c'est-à-dire des Aryens. Cette conception sera fortement critiquée par Sergi (1911, p. 114), néanmoins Stuhlmann (1912, p. 136) reprendra les mêmes conclusions. Deux ans plus tard, Guiffreda-Ruggeri (1914) fera preuve de prudence, en ne parlant que d'anonymes nordiques. Notons que pour Anton y Ferrandiz les blonds étaient plus exactement baltiques : "Es de advertir que en Marruecos, como en España, hay proporciones variables de la raza rubia baltica, bien visibles en el Rif, y de la morena alpina, de cabeza redonda... » (1903, p. 14).

9 Certains auteurs, cependant, pensaient que les blonds, pour être du Nord, n'étaient pas, pour autant, des baltiques ou des nordiques, Celtes, Germains ou Aryens. Bertholon, auteur avec Ernest Chantre des célèbres Recherches Anthropologiques dans la Berbérie orientale (1913), s'intéressait particulièrement à ce qu'il nommait «les Berbères de souche européenne » (1898). Il croyait initialement qu'il s'agissait de Celtes, puis crut qu'ils étaient Ligures (1888); il les découvrit enfin Danubiens (1893). Ces derniers seraient passés par l'Asie mineure et par l'Egypte pour arriver en Afrique du Nord. Afin d'appuyer sa démonstration, il utilise les sources égyptologiques, et particulièrement Rougé (1867) et Lenormant (1868), qui font état de Lybiens blonds qui envahirent l'Egypte entre 2000 et 1000 av. J.-C.

10 A l'instar de Faidherbe (1873), Bertholon ne cite les Lybiens blonds décrits par les Égyptiens que comme un moyen de datation de la migration des peuples nordiques; pour lui, cela ne signifie pas qu'ils étaient effectivement des Lybiens autochtones, sa théorie étant qu'ils furent Égéens et introduisirent la civilisation grecque en Afrique du Nord, comme pourrait en témoigner une étude de la culture kabyle (1913).

\section{Le paradigme des blonds autochtones}

11 La thèse de Shaw ne s'est toutefois pas seulement trouvée attaquée en ce qui concerne l'antériorité des blonds aux Vandales. C'est l'idée même d'une origine exogène de ceuxci, qui fut critiquée dès Gibbon (1985, t. II, p. 97), lequel voyait en eux des autochtones éclaircies par le climat de la montagne. Périer (1873) faisait également provenir les Berbères de l'Atlas, refusant - bien qu'il n'évoquât pas explicitement les blonds l'idée d'une origine étrangère des populations locales. Cette position sera ultérieurement celle de Sergi $(1895,1897,1901,1911)$, qui, reprenant les hypothèses de Hartmann $(1876,1880)$ et s'appuyant sur les travaux d'anthropologie militaire de Livi (1896), affirma que le blondisme n'est qu'un effet de l'altitude sur les populations méditerranéennes, observable aussi bien chez les Marocains de l'Atlas que chez les Italiens étudiés par Livi. Sergi développe cette argumentation dans The Mediterranean Race: A Study of the Origin of European Peoples (1901) : « If we turn to the region where the blonds in Africa are most numerous - that is to say, Morocco [...] There is therefore a region of perpetual snow, and a cold region constitued by valleys. May we not conclude that the same Libyan stock, established in North Africa from time immemorial [...] had undergone the diverse influences of external conditions of climate and soil forming variations in external physical characters ? [...] The centre of formation of the blond elements in North Africa would thus be in the Atlas valleys, especially in Morocco, and 
this would explain the fact that we find the greater number of blonds in this region. From this centre there would have been, inancient and succeedint times, a certain diffusion into the neighbouring countries* (Sergi, 1901, pp. 73-74).

L'intérêt de l'hypothèse de Sergi nous paraît résider, moins dans la détermination de la cause du blondisme, que dans la comparaison, qu'il opère, entre une population italienne et une population berbère, comparaison sous-tendue par l'idée de race méditerranéenne. Que des blonds apparaissent en altitude en Italie et au Maroc, n'a de sens que s'ils appartiennent à la même race ; c'est-à-dire s'il s'agit, au départ, d'une population brune qui offrirait une variante adaptative blonde. Weisgerber objecte à la théorie de Sergi que les blonds d'Italie pourraient tout aussi bien être les survivants d'une ancienne race blonde (1910, p. 127).

\section{La théorie de l'Atlantide}

13 Quand Périer (1873) parle d'Atlantes, qui constitueraient la population autochtone de l'Afrique septentrionale, cette dénomination désigne les habitants de l'Atlas. D'autres auteurs désignent par là les habitants de l'Atlantide; c'est de ces habitants que descendraient les blonds. Généralement, les considérations ayant trait à l'Atlantide passent pour peu sérieuses quoique prolifiques, Bessmerty (1949) ayant recensé plus de 2000 publications traitant de la question. Il parait cependant difficile de les méconnaître, dans la mesure où elles exercent un profond effet sur l'imaginaire du monde berbère, dont témoigne, par exemple, le roman de Pierre Benoit, L'Atlantide (1919).

14 Selon Berlioux (1883, p. 19), les blonds d'Afrique du Nord, seraient ethnogéniquement différents des bruns; les blonds descendraient des Atlantes alors que les bruns descendraient des Gétules sahariens. Les blonds ne seraient donc pas des Berbères, et, sous le nom de Lybiens, proviendraient, comme les Européens, de l'Atlantide. Cette idée est reprise par Levistre (1903, pp. 104-106), dans une longue communication à l'Académie d'Hippone; ce qui lui permet d'expliquer la présence des blonds antérieurement à l'invasion vandale, tout en contrant les théories aryennes.

15 Le problème de la répartition du type blond en Afrique du Nord a été clos par la publication de Kidder et al. (1955), après qu'ils eussent signalé des fréquences de blonds analogues dans bon nombre de populations méditerranéennes. L'hypothèse du phénomène adaptatif, telle qu'elle fut proposée par Sergi, s'avère séduisante, car elle pourrait expliquer partiellement la variation biologique observable entre différents groupes berbères. Elle a, cependant, été critiquée par Gsell, qui a fait remarquer que, si l'on trouvait des berbères blonds dans les pays montagneux tel le Rif, la Kabylie ou l'Aurès, ils paraissent être forts rares dans le Moyen et le Haut-Atlas, où l'altitude est plus élevée (1920, p. 308). Néanmoins, si l'on se réfère au concept d'isolat, et, a fortiori, au mécanisme de la dérive génique, tel qu'il a pu être observé par l'étude de la fréquence des groupes sanguins dans les populations berbères Aït Haddidou (Johnson et al., 1964) ou dans de familles juives des oasis du Tafilalet (Ikin et al, 1972), on peut envisager la possibilité d'une dynamique endogène du blondisme (Ki-Zerbo, 1980). Ceci implique que ces différences phénotypiques ne sont point opératoires dans l'établissement d'une classification raciale. 


\section{BIBLIOGRAPHIE}

Anton y Ferrandiz M., Razas y tribus de Marruecos, Madrid, Sucesores de Rivadeneyra, 1903.

Benoit P., L'Atlantide, Paris, A. Michel, 1919.

Berlioux E.-F., « Les Atlantes (Histoire de l'Atlantis et de l'Atlas primitif) », Annuaire de la Faculté de Lettres de Lyon, 1883.

Bertholon L., Esquisse de l'anthropologie criminelle des Tunisiens musulmans, Bibliothèque d'anthropologie criminelle et des sciences pénales, 1889.

Bertholon L., « Notice sur l'origine des berbères de souche européenne », Congrès de l'Association française pour l'avancement des sciences, Nantes, 1898, t. I, pp. 533-541.

Bertholon L., « Sociologie comparée des Achéens d'Homère et des Kabyles contemporains ", Revue tunisienne, 1913, n 97, pp. 190-199.

Bertholon L. et Chantre E., Recherches anthropologiques dans la Berbérie orientale, Tripolitaine, Tunisie, Algérie (Tome $1^{\text {er }}$ : Anthropométrie, craniologie, ethnologie), Lyon, A. Rey, 1913.

Bertrand A., " Monuments dits celtiques de la province de Constantine », Revue archéologique, 1863, (2 série), VIII, pp. 519-530.

Bessmertny A., L'Atlantide, Paris, Payot, 1949.

Broca P., « Les peuples blonds et les monuments mégalithiques dans l'Afrique septentrionale Les Vandales en Afrique ", Revue d'Anthropologie, 1876, t. V, pp. 393-404.

Buffon, Histoire naturelle de l'homme, t. II, Paris, Plassan, 1792 [1749].

Desmoulin A., Histoire naturelle des races humaines, Paris, Méquignon-Marvis, 1826.

Faidherbe (Gal), «Dolmens et hommes blonds de la Lybie », Matériaux pour l'histoire de l'homme, $1869,2^{\mathrm{e}}$ série, 7 , pp. 341-344.

Féraud L., " Monuments dits celtiques dans la province de Constantine », Bulletin de la société d'archéologie de Constantine, 1863, pp. 214-234.

Ferembach D., « Histoire raciale de l'Afrique du Nord », in Rassengeschichte der Menschheit, I. Schwidetzky Éd. München, Wien. Oldenbourg, 1975, pp. 90-142.

Gibbon E., Histoire du déclin et de la chute de l'empire romain, t. II : Byzance de 455 à 1500, trad. M.-P. Guizot, Paris, Laffont, 1985 [1788].

Gsell S., Histoire ancienne de l'Afrique du Nord, t. I, 1920 [1913].

Guiffrida-Ruggeri V., «Alcuni dati retrospettivi e attuali sulla antropologia della Libia », Archivio per l'antropologia e la ethnologia, 1914, XLIV, 1, pp. 255-288.

Hartmann R., Die Nigritier, Berlin, 1876.

Hartmann R., Les Peuples de l'Afrique, Paris, Germer Baillière, 1880.

Ikin E. et al, « The Blood groups and haemoglobins of the jews of the Tafilalet oases of Morocco ", Man, 1972, 7, pp. 595-600.

Johnson E., Ikin E., et Mourant A., « Blood groups of the Aït Haddidou Berbers of Morocco », Human Biology, 1963, t. 69, pp. 514-523. 
Kidder H.H., Coon C.S. and Briggs L.C., « Contribution à l'anthropologie des Kabyles », L'Anthropologie, 1955, t. 59, 1, pp. 62-79.

Ki-Zerbo J., «Théories relatives aux "races" et histoire de l'Afrique ", in L'histoire générale de l'Afrique, t. I, Méthodologie et histoire africaine, Paris, Jeune Afrique, UNESCO, 1980, pp. 291-299.

Kobelt W., Reiseerinnerungen aus Algerien und Tunis, Frankfurt am Main, M. Diest, 1885.

Lenormant F., Histoire ancienne de l'Orient, t. I, Les origines, les races et les langues, Paris, Levy, 1881.

Lenz O., Timbouctou (Voyage au Maroc, au Sahara et au Soudan), trad, de Lehautcourt, Paris, Hachette, 1886.

Leroi-Gourhan A. et Poirier J., Ethnologie de l'Union française, t.I, Afrique, Paris, P.U.F., 1953.

Levistre L., « Contribution aux études berbères », Bulletin de l'Académie d'Hippone, 1903, 30, pp.

25-114.

Lissauer A., « Archälogische und anthropogolische Studien über die Kabylen », Zeitschrift für Ethnologie, 1908, t. 40, pp. 501-535.

Livi L., Antropometria militare, Rome, D. Alighieri, 1896.

Maitrot Lt, « Arabes et Auvergnats », Recueil des notices et mémoires de la Société archéologique du département de Constantine, 1909, t. XII, $4^{\mathrm{e}}$ série, pp. 23-89.

Omalhius d'Alloy J.J. d', Des Races humaines (ou Eléments d'ethnographie), Paris, Lacroix et Baudry, 1859.

Pellat Ch., « Berbères : Histoire (Les Origines) », Encyclopédie de l'Islam, Leyden et Paris, Brill, Maisonneuve, 1960, p. 1208-1209.

Périer J.A.N., « Des races dites berbères et de leur ethnogénie », Mémoire de la Société

d'anthropologie de Paris, 1873, 2 ${ }^{\mathrm{e}}$ série, pp. 1-52.

Quedenfeldt M., « Eintheilung und Verbreintung der Berberbevölkerung im Marokko », Zeitschrift für Ethnologie, 1888, XX, pp. 98-130, 146-160, 184-210.

Rougé de, «Extraits d'un mémoire sur les attaques dirigées contre l'Égypte », Revue archéologique, 1867, XVI, pp. 35-82.

Saint-Rémy G., « Anthropologie et ethnographie, II, Afrique du Nord », Bulletin de la société de géographie de l'Est, 1904, 2, pp. 1-15.

Sergi G., Origine e diffusione della stirpe mediterranea, Rome, D. Alighieri, 1895.

Sergi G., Africa (Antropologia della stirpe camitica), Turin, Bocca, 1897.

Sergi G., The Mediterranean Race : a Study of the Origine of European Peoples, Londres, W. Scott, 1901.

Sergi G., L’Uomo, Turin, Bocca, 1911.

Shaller W., Esquisse de l'État d'Alger, trad. X. Bianchi, Paris, Ladvocat, 1830.

Shaw T., Voyage dans la régence d'Alger (ou Description géographique, physique, philologique, etc. de cet état), trad. J.Mac Carthy, Paris, Marlin, 1830 [1738].

Stuhlmann F., Ein Kulturgeschichtlicher Ausflug in den Aures (Atlas von Süd-Algerien), Hambourg, Friederischen \& Co., 1912.

Topinard L., « Les Ossements de Spy et l'ethnographie de la Tunisie, Rapport sur le concours du prix Broca », Bulletins de la Société d'anthropologie de Paris, 1889. 
Urvoy de Closmadeux J., « Conférence sur l'origine des Berbères », Revue tunisienne, 1897, pp. 465-472.

Vallois H., Les Races humaines, Paris, P.U.F., 1944.

Vivien de Saint-Martin L., Le nord de l'Afrique dans l'Antiquité, Paris, Imprimerie impériale, 1863.

Weisgerber H., Les Blancs d'Afrique, Paris, Doin, 1910.

INDEX

Mots-clés : Antiquité, Histoire 